\title{
POI Explorer - A Sonified Mobile Application Aiding the Visually Impaired in Urban Navigation
}

\author{
Piotr Skulimowski, Piotr Korbel and Piotr Wawrzyniak \\ Institute of Electronics \\ Lodz University of Technology \\ Lodz, Poland \\ piotr.skulimowski@p.lodz.pl,piotr.korbel@p.lodz.pl,piotr.wawrzyniak@dokt.p.lodz.pl
}

\begin{abstract}
The paper presents POI Explorer mobile application aiding the visually impaired in spatial orientation and in urban navigation. A user equipped with a smartphone with accelerometer, electronic compass, mobile data transmission and positioning capabilities can access information on nearby points of interest. Maintaining data connection with dedicated system servers provides access to additional services facilitating the navigation in urban areas. The paper describes an overall architecture of the system. Then, the details of the user interface of the application are presented. The user interface of the application was designed to meet both the needs of visually impaired users and the requirements imposed by dynamic data changes. It employs a unique combination of text-to-speech and sonification techniques to ensure clarity of messages as well as high responsiveness of the application. The results of experiments performed in areas with different densities of points of interest proved the usability of the proposed approach.
\end{abstract}

Index terms-Location-based services, mobile computing, pervasive computing, electronic aids, visually impaired

\section{INTRODUCTION}

$\mathrm{R}$ ECENTLY, a number of electronic travel aids (ETA) addressing the needs of the visually impaired have been developed [1-9]. Such systems can be used to overcome difficulties with spatial and geographical orientation and navigation, and facilitate access to various public services. Those difficulties become especially cumbersome in urban areas. Lack of good spatial orientation makes difficult to find a safe path among obstacles, and to locate and identify points of interest (POI) like bus stops, offices, restaurants, or even pedestrian crossings [1].

Dedicated electronic travel aids play a vital role in aiding the visually impaired in everyday activities. Such assistive devices are usually equipped with a GPS receiver to provide precise information on user terminal position, GSM/UMTS transceivers, inertial sensors, and a speech synthesizer enabling auditory form of presentation of various data to the user [2, 4, $8-9]$. Such devices can usually be carried in a pocket $[2,4,9]$ and because of dedicated user interfaces (tactile keyboards, text-to-speech and speech-to-text systems) are easy to use by visually impaired users $[2,4,9]$. Most of dedicated ETAs allow

This work was partially supported by the National Centre for Research and Development of Poland under grant no. NR-02 0083-10 in years 20102013. to lead visually impaired or blind users along previously recorded routes. They also store databases of POIs, which can serve as an aid in orientation. Due to the fact that such devices are produced only in short series, the build quality and functionality of such solutions may be far from that of massively produced electronic devices. The main disadvantages limiting the popularity of such systems include difficulties in upgrading software and high unit costs.

With the growth of popularity of advanced mobile phones, more and more applications aiding the visually impaired in navigation and travelling appear on the market [10-17]. Modern phones are usually equipped with advanced positioning capabilities as well as in a range of additional sensors, like accelerometers, gyroscopes or magnetometers. ETA solutions using mobile phones as user terminals may benefit from the use of detailed digital maps from different providers, like Google Maps, Bing Maps, OpenStreetMap, etc. in combination with satellite (GPS, Glonass) or network (cellular, Wi-Fi) based positioning techniques. Also, mobile phones usually are equipped with good quality speech synthesizers facilitating communication with visually impaired users.

Since most of the users use the built-in text-to-speech and sonification systems, such as VoiceOver [18] or TalkBack [19], navigation applications are adapted to work with these systems. For this reason, application user interfaces use standard system components like buttons, text boxes, lists, etc. The drawback of such an approach is the lack of adaptation of very small items (which you must specify for the sonification) to the screen reader systems, or long, scrollable lists with dynamic contents.

This article describes a novel approach to presenting information about the POIs using combination of text-tospeech and sonification techniques. The remainder of the paper is organized as follows. Section II provides an overview of the system architecture while Section III gives details of the POI Explorer mobile application functionality. A proposed approach to auditory presentation of POI data is described in Section IV, and the results of application tests are presented in Section V. Finally, Section VI summarizes our work.

\section{SYSTEM ARCHITECTURE}

The architecture of the proposed system for guidance of visually impaired in urban environment is shown in Fig. 1. The system consists of two subsystems: application servers and mobile user terminals with dedicated applications. 
The mobile user terminal is an Android OS based smartphone equipped with a dedicated POI Explorer mobile application. Since the application uses Sensors API of the operating system, it can be run on devices with Android OS in version 2.3 or newer. The details of the application are provided in Section III.
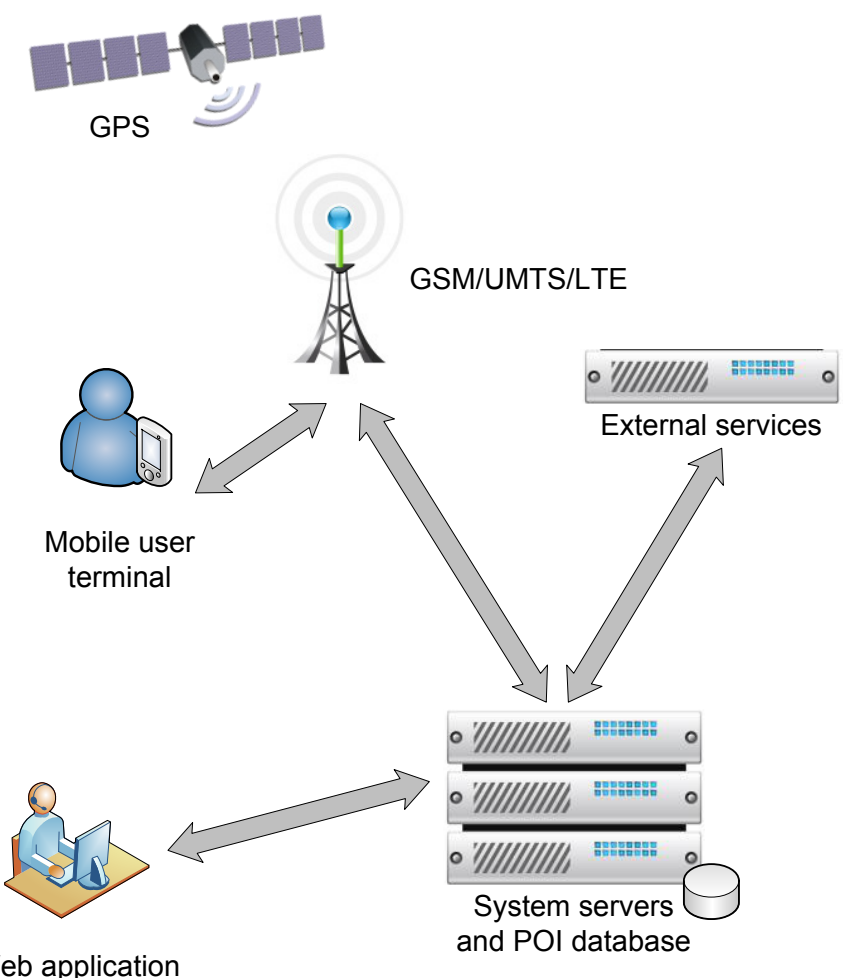

Fig. 1. Architecture of the electronic system for guidance of the visually impaired in urban environment.

The main function of the system server is storing a database of points of interest. The prototype solution uses MySQL relational database management system. POIs are organized into categories and subcategories, what allows to find necessary information easily. The POIs stored in the database can be both public and private. The public POIs are available to all the users of the system, while the private ones are accessible only to the owner. Users can also add additional personalized information to the points (text notes, voice recordings) to enrich the database.

The server also hosts web application for remote POI data management. The web based application (Fig. 2, Fig. 3) uses PHP and AJAX and is dedicated for sighted users assisting the visually impaired and blind. The management application allows to add and remove points of interest. It is also possible to define new categories of the POIs as well as hierarchy of the different categories of points. An important functionality of the system is the ability to define routes which the visually impaired can follow. This may be for example a route leading to the office, shop, etc. The routes can be created in one of two ways: from the POI Explorer mobile application and from the aforementioned web based management application. Examples of such predefined routes are shown in Fig. 2. The management application allows also to delete or change the order of the points in a route.

The data are exchanged between the POI Explorer mobile application and the system server in an XML format. Examples of XML system messages are presented in Fig. 4 and Fig. 5. Hierarchical structure of XML documents allows elastic and extensible POI category management. Moreover, such a solution allows to provide a universal application programming interfaces (API) to other, external platforms. For example, it is possible to import waypoints and routes from external sources like Loadstone database [11].

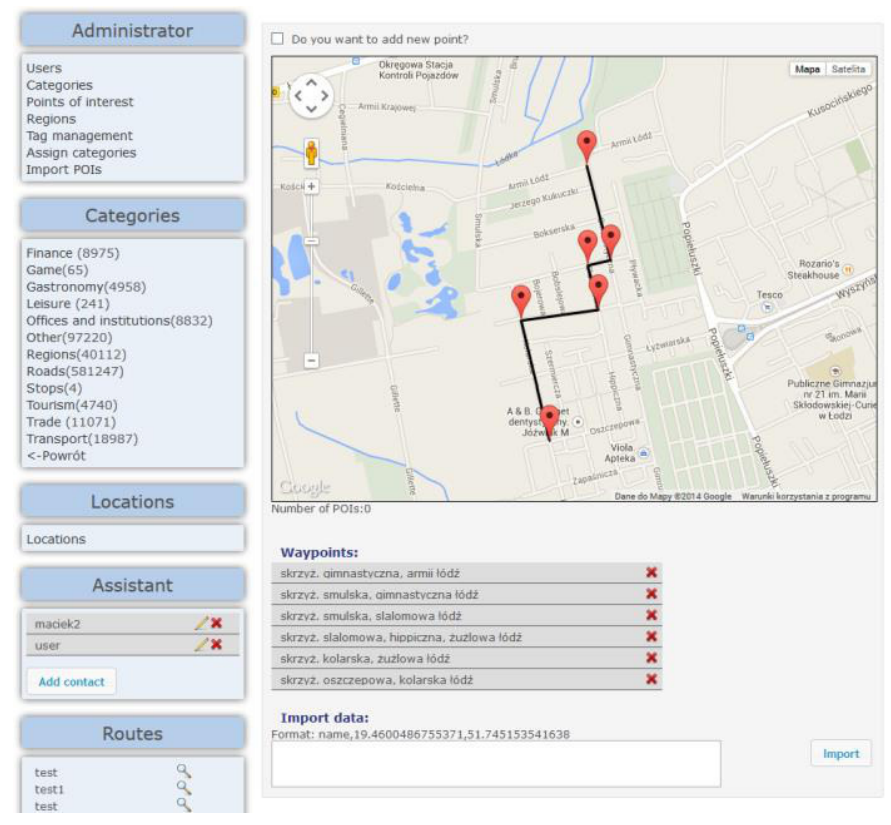

Fig. 2. The graphical user interface of a web application for the management of the POI database - path creation example.

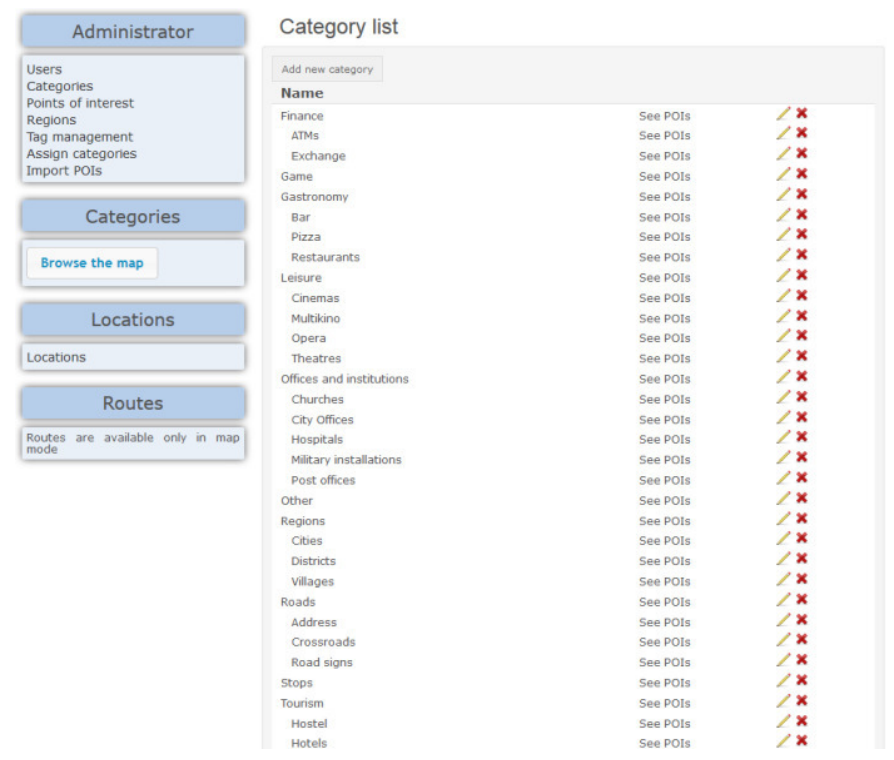

Fig. 3. The web application for the management of the POI database. The categories and subcategories of POIs are shown, each of the database entries can be edited or deleted. 


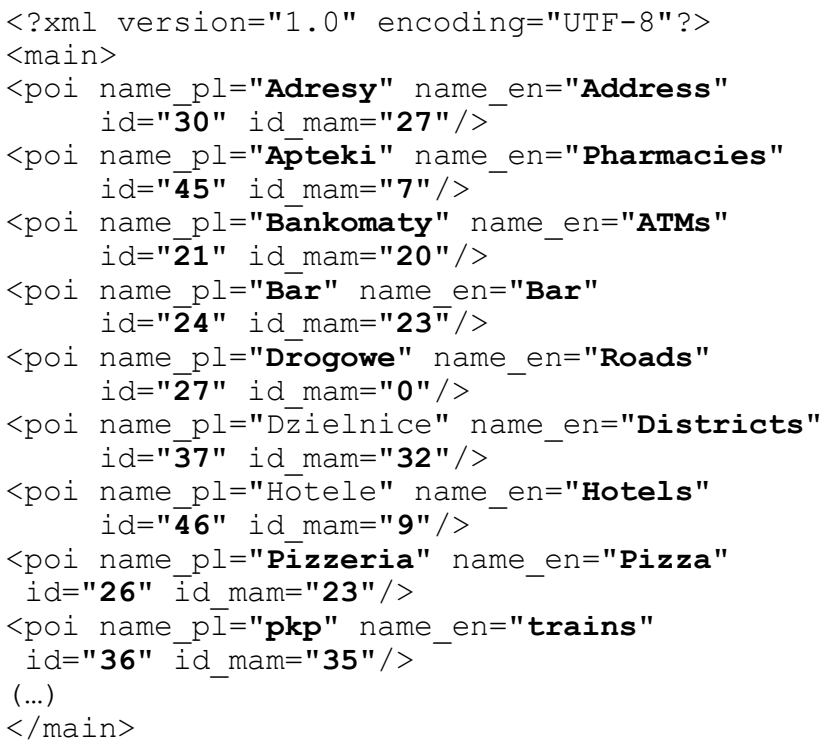

Fig. 4. The example XML server response contains list of categories. Each category has its name in Polish and English language, identification number and identification number of parent category.

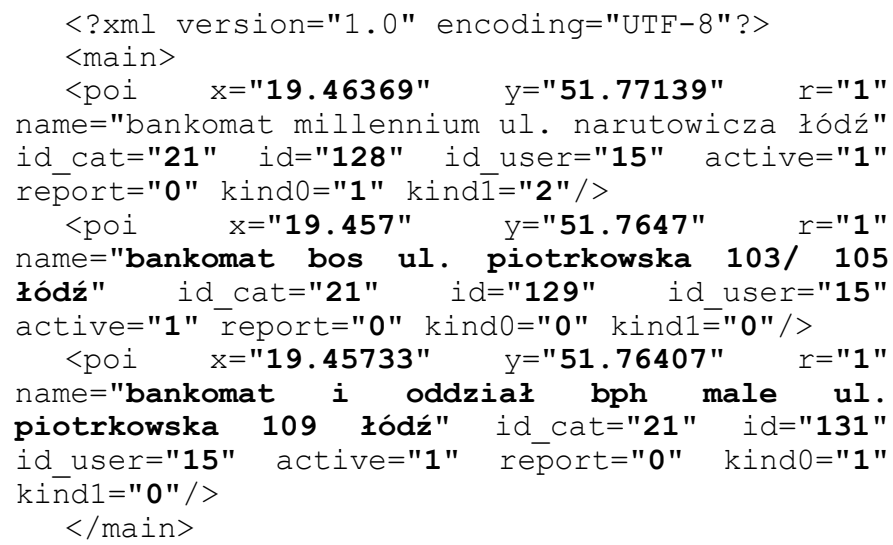

Fig. 5. The example XML server response contains list of points in the given area. Each of the point of interest has GPS cooridnates, radius $r$ in meters, name, category id (id_cat), point id (id), id of user who added this point (id_user), active status (active), number of reported bugs (report), number of text messages (kind0) and voice notes (kind1).

\section{SMARTPHONE BASED URBAN NAVIGATION - POI EXPLORER}

The first version of POI Explorer application aiding the visually impaired in moving in the urban area was developed for Symbian OS based mobile phones [20]. As Symbian based devices no longer play an important role on the market, the application had to be migrated to a new platform.

Nowadays iOS and Android based mobile phones are of a special interest for blind and visually impaired users. The reason for that is that both the systems offer built-in and well integrated with the operating system text-to-speech modules: Voice Over (iOS) [18] and TalkBack (Android) [19]. Availability of such high quality system modules allows developers to create their own applications using standard GUI elements which can be easily presented to the visually impaired users. Moreover, most of contemporary smartphones are equipped with touch screens and offer gesture-based screen readers supported by multitouch capabilities. For example moving a single finger over the list causes a list item to be read, while moving of two fingers can be used to scroll the list.

After market analysis and discussions with representatives of the target group of POI Explorer users, we have decided to select Android Operating System based devices as a target platform. According to ABI Research Android is the most popular operating system (77\% share in Q4 2013) [21]. In 2013, according to Gartner Android market share was 78,4\%, while iOS $15.6 \%$ [22]. Another advantages include lower costs of Android phones in comparison to iPhones as well as availability of more accessible low-end devices.

The development of the application was based on test results of previous releases for Symbian based mobile phones as well as initial releases for Android OS based devices. About 20 blind and visually impaired volunteers received the aforementioned applications for testing purposes. The volunteers from different regions of Poland were recruited through an advertisements on Loadstone [11] mailing lists. Although now deprecated, at that time Loadstone was one of most widely used applications for navigation of the blind and visually impaired. The users of competitive application positively rated the idea to use a compass to determine the direction of movements (instead of analyzing history of GPS values). There have been requests for the adding information on the number of satellites used to calculate GPS coordinates, which allows them to rate, how much they can trust the messages returned by the application. There were different opinions about the possibility of downloading lists of POIs around the current position of the user. On one hand, a visit to another city does not require prior preparation of a list of points of interest, on the other hand there were concerns that data for some reason would not be downloaded. There were a lot of positive comments about organizing POIs into categories and subcategories, because it allows to reduce the number of points to be presented to the user.

Graphical user interface layout and functionality of POI Explorer mobile application have additionally been consulted with blind and visually impaired users from the Polish Association of the Blind. As the result, the graphical user interface of POI Explorer (Fig. 6, Fig. 7, Fig. 9, Fig. 11, Fig. 14) uses large, high contrast characters aiding users with moderate visual impairment. Also, system requirements of our applications allow to run them on low cost devices.

To use the application, the user needs to set up his/her system account. This is an optional step, however it allows to store private points of interests and routes. It also enables the possibility to adding notes and voice description to POIs. When the user runs the application for the first time, a list of POI categories is downloaded from the server. Moreover, for a selected area (the user can define its radius) a range of POIs are downloaded and stored in local database. It allows the use of the application without maintaining Internet connection. 


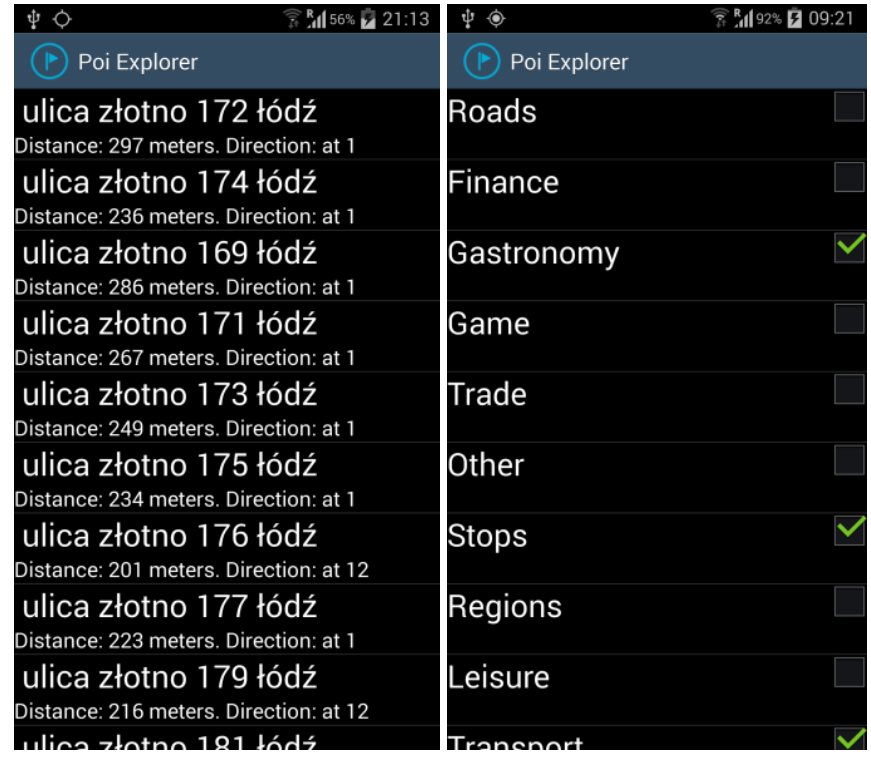

Fig. 6. Screenshots from the POI Explorer application captured by Samsung Galaxy S4 Android Phone with KitKat 4.4.2. The list of the points of interests in the front of the user (direction is calculated based on compass values) are presented in a standard listbox. On the right: list of categories, each of them can have unlimited number of subcategories.

The POI Explorer supports the navigation of the blind users in one of three different modes. The users can choose navigation: along a predefined path, to the selected point (e.g. a bus stop), or in a "look around" mode. The distance to a given POI is calculated based on readings from the built-in GPS receiver, while orientation in the area is calculated on the basis of the values returned by the compass module. The data for the user are being refreshed on demand and take into account the current readings of the two aforementioned sensors.

\section{A. Navigation to the specific point}

In this mode, the user picks from a list of points of interest any entry. Then the user receives the information about the distance of this point from the current position and direction to the point expressed in the "per hour" mode. Orientation is determined relative to the current position of the phone and the point. POI Explorer calculates the angle between the orientation of the phone (from the compass) and the orientation of the target point, which is then converted to the appropriate "hour". Example message is shown in Fig. 7. In addition, the user can designate the route to the point using Google Maps mechanism, which returns a list of waypoints.
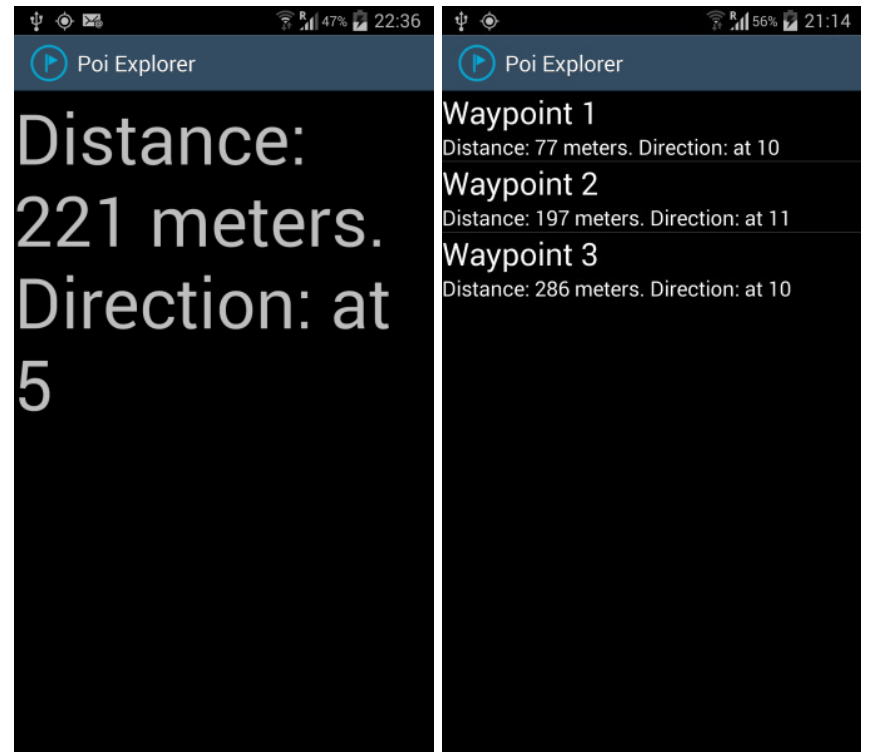

Fig. 7. Screenshots from the POI Explorer application captured by Samsung Galaxy S4 Android Phone with KitKat 4.4.2. On the left: mode distance/direction, on the right: list of waypoints obtained from the Google Maps engine.

\section{B. Navigation along a recorded path}

User have possibility to register the route and determine intermediate points like intersections, characteristic points etc. Example of such route is shown in Fig. 2. The route most often is created with an assistance of a sighted person. The user is guided to the each of the waypoints as described in section IIIA. When the waypoint is reached, POI Explorer changes the destination point to the next one from the list. This mode is most liked by the target group of users due to the possibility of creating waypoints in a supervised way.

Automatic determination of intermediate points is also possible. In this case, there is a risk that the algorithm will not take into account slight changes in direction of the route. Moreover, in this mode it is not possible to automatically add information on dangerous locations, such as defects in the roadway, etc.

\section{Look around mode}

Look around mode is one of the most important features of POI Explorer especially useful in unfamiliar locations, for example, in an unknown city. A list of points of interest within a certain radius with the indication of the distance and direction is presented to the user. The blind person can restrict presented points only to selected categories. An example of such filtered list is shown in Fig. 6.

POI Explorer was written taking into account the suggestion from the target group of users. Some application capabilities have been developed as the result of requests from our testers. These for example include the use of a compass to determine direction (usually applications determine direction based on the history of GPS values) or importing data from the Loadstone, very popular platform aiding blind and visual impaired people for Symbian OS [11]. 
Unfortunately, interaction with a touchscreen of a smartphone requires the use of both hands and may be especially uncomfortable for blind users, who at the same time are often using a white cane. Moreover, screenreaders are not very convenient for sonification of dynamic GUI controls.

The tests with the target group of users revealed that the main problem for them is the way of sonification by built-in screenreaders. Every change in the user interface (for example resulting from an update of a text field, or from appearance of a new item in the list) implies that the user is informed about that change. Finding the selected POI often requires reading of the entire list of points (Fig. 6). Any update of the interface contents may interrupt reading of the items from the list and force re-reading of the entire information about the screen elements. In addition to that, every change of position (due to changing user's position or fluctuations in GPS or compass readouts) causes, that the list frequently changes its contents. Moreover, because the list is sorted by distance from the user, the order of the points in the list can also change. The user is notified by the screen reader on every such change. Excessive information becomes hardly understandable and confusing for the user. This problem has become an inspiration to propose another approach to presentation of information on points of interest located in the vicinity of the user.

\section{SONIFIED PRESENTATION OF INFORMATION ON NEARBY POIs}

When designing the new method of presentation points of interest, to avoid the previously described problems, the following assumptions have been made:

- the usage of the application interface should be possible with only one hand. Due to the safety of the blind, it is advisable that the phone should be hidden in a pocket, and voice messages could be transmitted via the handsfree set;

- amount of conveyed information should be limited to the minimum, and the number of voice messages should also be reduced. Previously conducted experiments showed that the excess of voice messages is very tiresome and discourage users to use such kind of applications;

- it should be possible to reduce the number of points from the vicinity presented to the user at a single time;

- a way to read detailed information about the point being currently sonified should be provided.

To fulfill of all of the above requirements, it was decided that a unique short sound will be assigned to the each category of the POIs. The points will be presented to the blind person in the order of increasing distance from the user. Only the points located along a straight line (within a predefined angle) from the user will be presented.

After starting of the scan, a short audio message is played. A virtual circle of search is moving away from the user and when it encounters a POI on its way, a sound associated with given category of the point is played. After completion of playing the sound, the radius of the circle of search is increased until it reaches the next point from the user vicinity (Fig. 9, 11). Fig. 9 and 11 show application screens displaying the POIs found in the user vicinity, while Fig. 8 and 10 show these points on the Google Map.

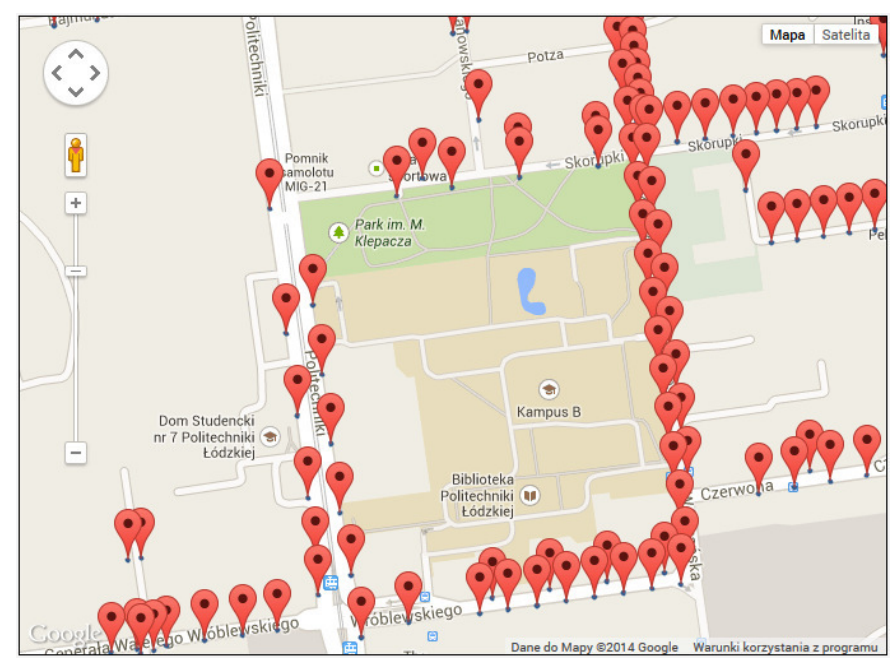

Fig. 8. POIs from the selected category Address shown on the Google Maps (screenshot from the web application). Number of POIs presented to the user is limited to 100. A fragment of the map corresponds to the area shown in Figure 9.

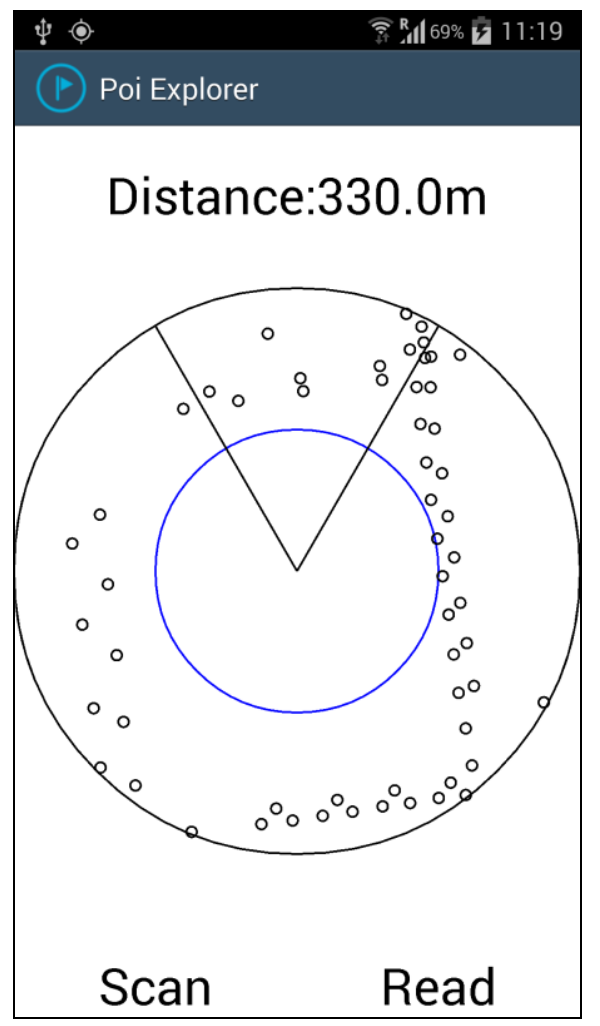

Fig. 9. Screenshot from the POI Explorer application captured by Samsung Galaxy S4 Android Phone with KitKat 4.4.2. Each point of interest from Figure 8 is marked with a circle. 


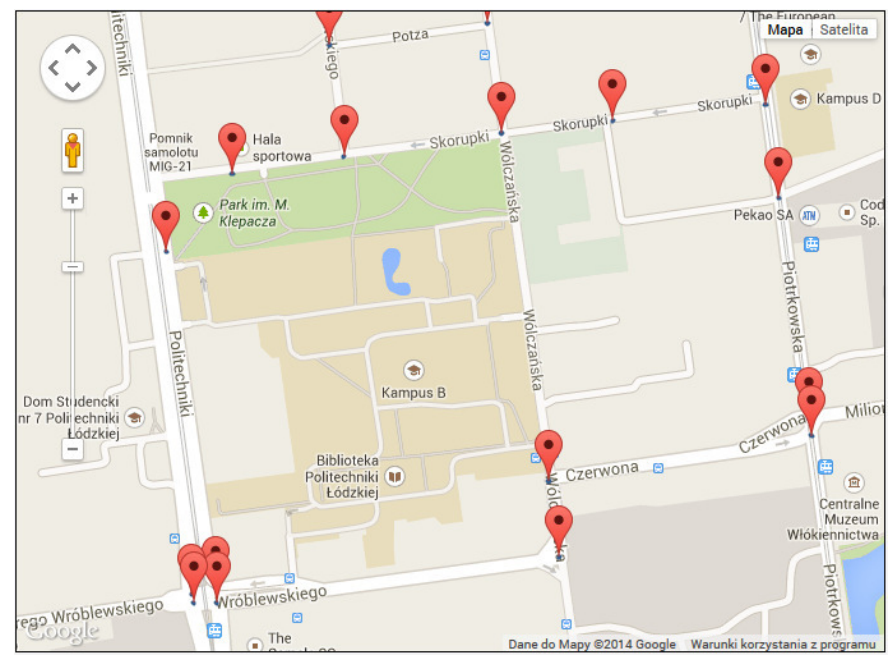

Fig. 10. POIs from the selected category Intersection shown on the Google Maps (screenshot from the web application). A fragment of the map corresponds to the area shown in Figure 11.

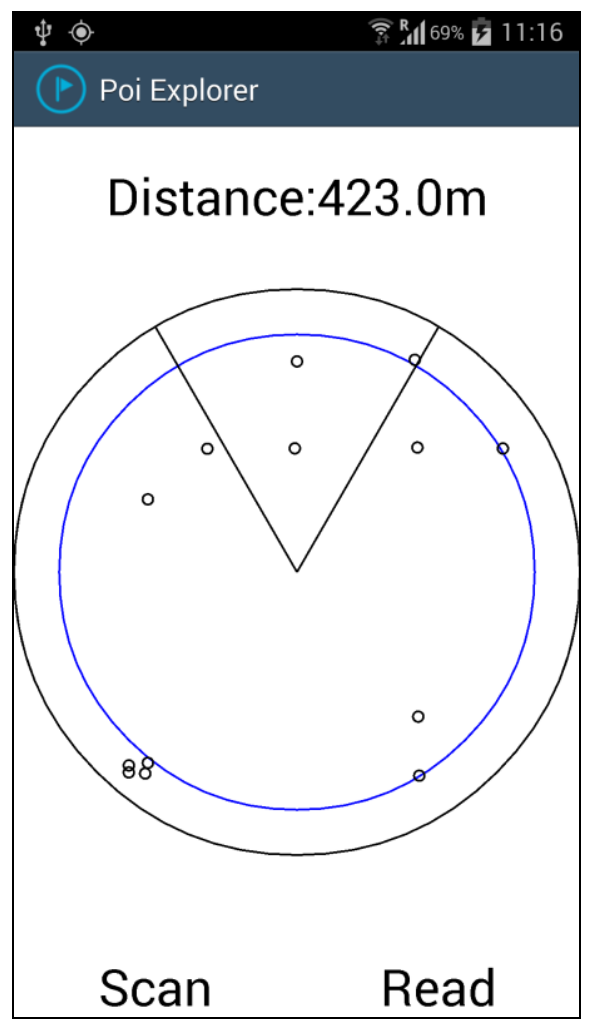

Fig. 11. Screenshot from the POI Explorer application captured by Samsung Galaxy S4 Android Phone with KitKat 4.4.2. Each point of interest from Figure 10 is marked with a circle.

Preliminary tests performed at early development stages revealed that updating the positions of points during the scan often causes confusion, because the scanning time strongly depends on the number of POIs and can take up to several seconds. For this reason, it is assumed that update of the positions of POIs in relation to actual user position occurs at the beginning of the scan.
In the application for navigation purposes we set out three control fields. The field at the top allows the user to change the scan range. The higher the $\mathrm{x}$ coordinate of a touch point, the larger the scan area. Change (releasing) the touch location on the screen sets the new value of the radius of the scan, the selected value is read to the user. Scan area at the bottom of the screen causes that scan starts from the beginning. It may be useful at a time when the user changes its orientation and wants to start scanning points after update their relative position. Field Read allows to read information about the recently sonified point. When the user selects this option, the distance of the point and its orientation relative to the user is read.

Internal tests have shown the system accuracy decreases with the growth of the number of points to be presented. An example of such a situation is shown in Fig. 9. Points arranged in lines represent neighboring buildings along the street. Fig. 8 shows the presented area on the Google Map (only addresses and intersections are shown).

To make it easier to read the information about points we introduced manual mode, which uses the readouts from the accelerometer to control the presentation of the POI list (Fig. 12).
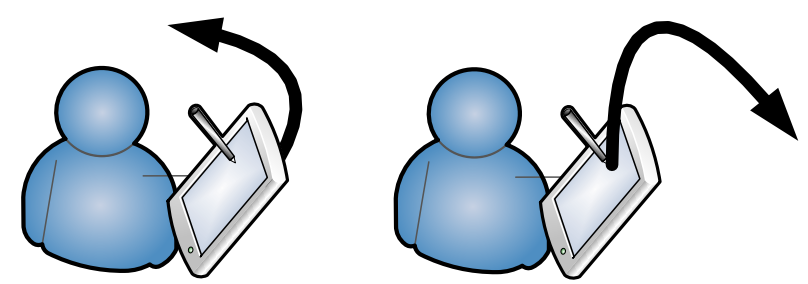

Fig. 12. Gesture based control of the device - Move to the previous POI (left), Move to the next POI (right).

A move of the phone downwards stops scanning and information about the next point on the list is read. Contrary, the upward gesture starts reading of the information about the previous point. The threshold values of data from the accelerometer readings were chosen to eliminate false gesture detection. Our solution allows to manipulate the phone hidden in the jacket pocket. At any time it is possible to finish manual mode and start automatic scanning.

A. The use of built-in motion sensors for gesture recognition

Motion sensors: gyroscope, accelerometer, gravity sensor, linear acceleration sensor can be utilized in finding user gesture patterns. They measure acceleration forces and rotational forces along three axes. Some of them are hardware-based, while others are software-based: they derive data from one or more hardware sensors. Accelerometer sensor measures acceleration force in SI units that is applied to the device on all physical axes. The values include the force of gravity. Gravity sensor (which can be a software sensor) measures only the force of gravity. Gyroscope returns device's rate of rotation around each of the three axes. Linear acceleration works like the acceleration sensor, but values exclude the force of gravity. Not all the sensors are available on every device. For example gravity sensor is not available on devices with API versions lower than 9. Android allows to determine the capabilities of sensors, like maximum range, resolution etc. These values can 
differ between devices. It is also possible to get notifications on accuracy changes or when a sensor reports a new value. Each log file entry also contains a timestamp [23].

Block diagram of accelerometer based gesture recognition is shown in Fig. 13. All values (x, y, z components) returned by accelerometer are in SI units. Because a sensor measures acceleration applied to the device, the values returned by the device include gravitational acceleration. During gesture detection we take into consideration only values of $y$ component. Initially, we expect to begin a gesture. It is a condition in which the user is holding the phone horizontally $\left(|y|<T h h_{y 1}\right)$. Then we expect to perform the gesture. The user must tilt the phone up or down. If $\left(|y|>T h_{y 2}\right)$ an appropriate action is performed. To detect another gesture, the phone should return to the horizontal position. The algorithm works only when the gravitational acceleration is only applied to the device. All values not satisfying criteria (1) are ignored. During the tests with Samsung Galaxy S4 device, the $\mathrm{Th}_{\mathrm{y} 1}$ and $\mathrm{Th}_{\mathrm{y} 2}$ threshold values were set to 2 and 5 correspondingly.

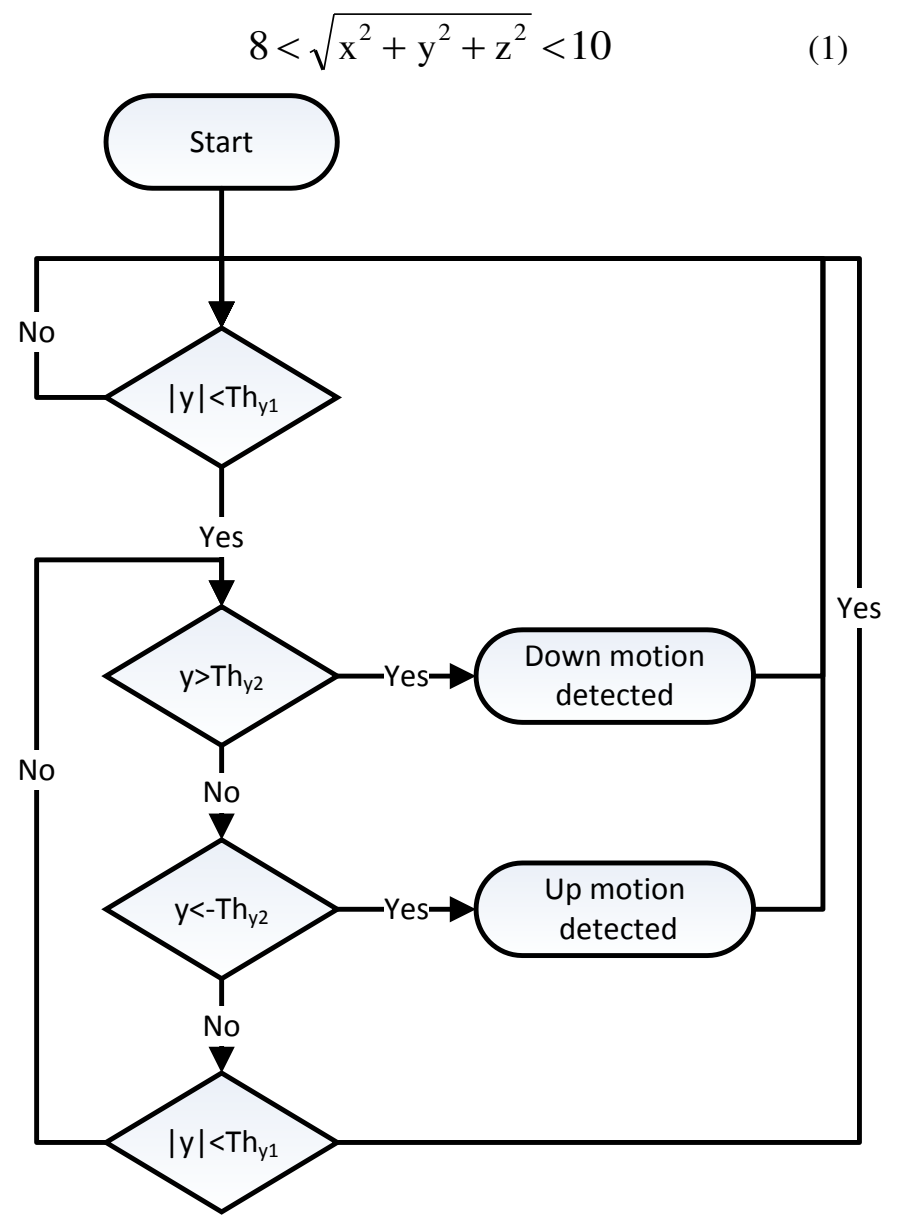

Fig. 13. Accelerometer based gesture recognition - block diagram of the algorithm.

\section{APPLICATION TESTS}

Application tests were carried out in selected areas of the city of Lodz, in places containing a large number of points of interest. As a test platform we have chosen Android 4.2 and 4.4.2. Fig. 14 shows POI Explorer running on Samsung Galaxy S4 mobile phone. A group of several sighted volunteers at different age and with different familiarity of mobile technologies was involved in the tests.

An option to specify the radius of the scan turned out to be a good solution. It allows to eliminate the problem of too high number of points to be presented at the time. Accelerometerbased control worked properly, there were no problems with reading information about the points in the vicinity. Testers pointed out that despite the possibility to specify the categories of presented points, there should be an option to hide unnecessary points.

During the tests of the application, time of a complete scan was set to about 15 seconds. In case of several points in the vicinity, it was the optimal time for selecting the chosen point of interest by the user.

The active fields (the slider for setting the distance, Scan and Read buttons) in the GUI seem to be well matched. We did not notice problems in locating these fields, even while the phone was kept in the user's pocket.

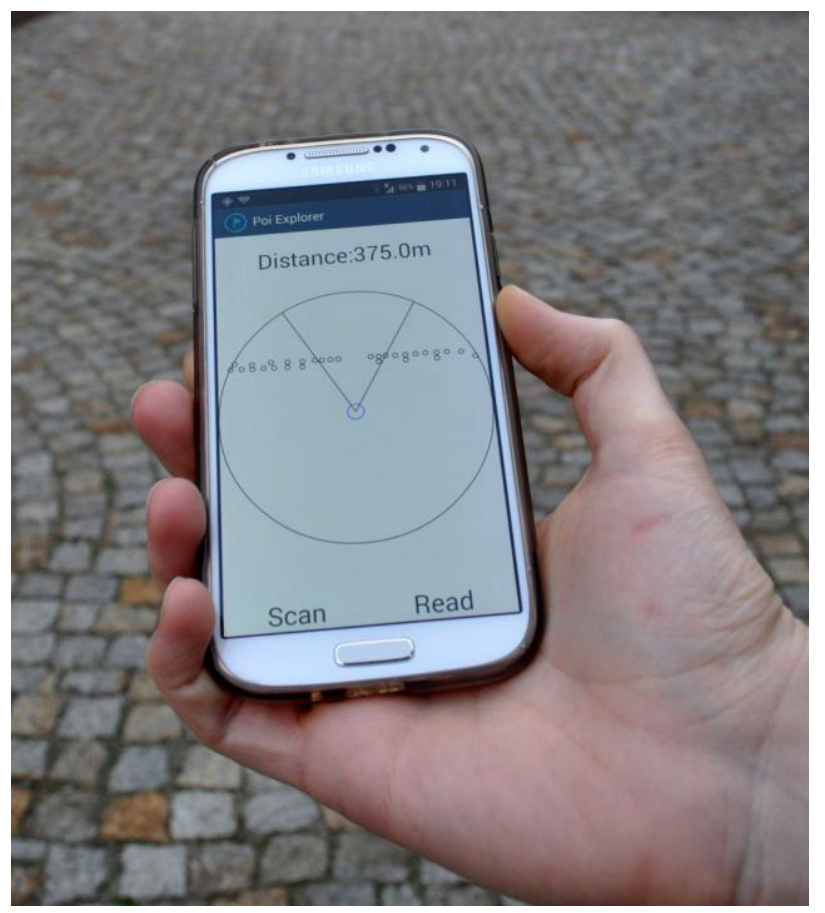

Fig. 14. POI Explorer launched on Samsung Galaxy S4 Android KitKat phone.

The tests of the application revealed the limitations of audio playback system on Android OS. Playing short audio samples indicating the points ran smoothly. The system allows to play several audio samples at the same time. Playing a voice message interrupts the previous message. Fortunately, it is possible to play sounds when reading messages by Text-ToSpeech engine.

Since most of contemporary mobile phones are equipped with electronic compasses, these can be kept in a pocket and the user can use the compass readouts for the orientation 
purposes. Text to speech TalkBack service of the phone can be used to read system messages and POI descriptions to the user.

\section{SUMMARY}

Although many electronic travel aids have been developed so far, urban areas still remain hardly accessible to the visually impaired. Poor spatial orientation and inability to access textual information makes difficult to move around the cities. Development and growing popularity of new generations of advanced mobile devices opens up new opportunities to create inexpensive and efficient ETAs for the blind and visually impaired. Even inexpensive mobile phones are nowadays often equipped with reasonably good quality sensors (compass, accelerometer, A-GPS receiver). In addition, speech synthesis, text-to-speech and speech recognition systems allow to offer alternative communication interfaces to visually impaired users. Using general purpose mobile devices as platforms for development of ETAs lowers deployment costs of such systems and as a result the user can carry around only a single device instead of a range of devices dedicated to different purposes. Also cloud computing and storage solutions provide higher security and facilitate access to user data in the case of a change of the device.

The system described in the paper is primarily intended to aid the visually impaired in urban navigation. It uses a novel approach to presenting information about the points of interest in the vicinity of the user. The combination of text-to-speech and sonification techniques allows to effectively reduce the amount of excessive information presented to the user and overcome the limitations of popular screen reader systems.

Currently, our efforts are focused on preparation of the release candidate version of the application, that will be made available to a wider group of blind and visually impaired volunteer testers.

\section{ACKNOWLEDGMENT}

This work was partially supported by the National Centre for Research and Development of Poland under grant no. NR02 0083-10 in years 2010-2013.

\section{REFERENCES}

[1] P. Strumiłło, "Electronic Interfaces Aiding the Visually Impaired in Environmental Access, Mobility and Navigation," Proc. 3rd International Conference on Human System Interaction, Rzeszów, Poland, 2010, pp. 17-24, http://dx.doi.org/10.1109/HSI.2010.5514595

[2] Kapten Mobility. http://www.kapsys.com/fr/en/products/kaptenmobility/. Accessed 22 April 2014.

[3] Talking Signs. http://www.talkingsigns.com/. Accessed 22 April 2014.

[4] Trekker Breeze handheld talking GPS, http://www.humanware.com. Accessed 22 April 2014
[5] J. Marski, P. Bajurko, K. Radecki, and T. Buczkowski, „Miniaturowe radiolatarnie $\mathrm{i}$ terminale $\mathrm{z}$ sygnalizacja $\mathrm{RSSI}$ do wspomagania orientacji osób niewidomych," Przegląd Telekomunikacyjny i Wiadomości Telekomunikacyjne, 6, 2010, pp.320-323. (in Polish)

[6] PAVIP. http://bones.ch/. Accessed 22 April 2014.

[7] Step-Hear. http://www.step-hear.com/. Accessed 22 April 2014.

[8] P. Barański, M. Polańczyk, P. Strumiłło, “A Remote Guidance System for the Blind," Proc. 12th IEEE International Conference on e-Health Networking, Applications and Services HealthCom, Lyon, France, 2010, http://dx.doi.org/10.1109/HEALTH.2010.5556539

[9] NaviEye (Nawigator). http://www.migraf.pl/. Accessed 22 April 2014.

[10] Ł. Kamiński, K. Bruniecki, "Mobile Navigation System for Visually Impaired Users in the Urban Environment," Metrology and Measurement Systems XIX (2), 2012, pp.245-256.

[11] Loadstone GPS - Free GPS Software for Your Mobile Phone. http://www.loadstone-gps.com/. Accessed 22 April 2014.

[12] P. Korbel, P. Skulimowski, and P. Wasilewski, "A Radio Network for Guidance and Public Transport Assistance of the Visually Impaired," Proc. 6th International Conference on Human System Interaction HSI 2013, Sopot, Poland, 2013, http://dx.doi.org/ 10.1109/HSI.2013.6577869

[13] P. Korbel, P. Skulimowski, P. Wasilewski, P. Wawrzyniak, "Mobile applications aiding the visually impaired in travelling with public transport," Computer Science and Information Systems (FedCSIS), 2013 Federated Conference on , vol., no., pp.825-828, 8-11 Sept. 2013.

[14] P. Eslambolchilar, A. Crossan, R. Murray-Smith, "Model-based target sonification on mobile devices," Proceedings of International Workshop on Interactive Sonification, 8 January 2004, Bielefeld, Germany, 2004.

[15] B. Taylor, Dah-Jye Lee, Dong Zhang, Guangming Xiong, "Smart phone-based Indoor guidance system for the visually impaired," Control Automation Robotics \& Vision (ICARCV), 2012 12th International Conference on, pp.871-876, 5-7 Dec. 2012, http://dx.doi.org/10.1109/ICARCV.2012.6485272

[16] K. Koiner H. Elmiligi, F. Gebali, "GPS Waypoint Application," Broadband, Wireless Computing, Communication and Applications (BWCCA), 2012 Seventh International Conference on, vol., no., pp.397,401, 12-14 Nov. 2012, http://dx.doi.org/10.1109/BWCCA.2012.71

[17] Wang Guolu; Qiu Kaijin; Xu hai; Chen Yao, "The design and implementation of a gravity sensor-based mobile phone for the blind," Software Engineering and Service Science (ICSESS), 2013 4th IEEE International Conference on, pp.570-574, 23-25 May 2013, http://dx.doi.org/10.1109/ICSESS.2013.6615373

[18] VoiceOver. http://www.apple.com/accessibility/voiceover/. Accessed 22 April 2014.

[19] Google TalkBack, https://play.google.com/store/apps/details?id= com.google.android.marvin.talkback. Accessed 22 April 2014.

[20] M. Polańczyk, P. Skulimowski, B. Sujecki, and D. Sulmowski, "Personal Navigation System for the Blind based on Points of Interest," Proc. II Forum Innowacji Młodych Badaczy 2011, Łódź, Poland, 2011.

[21] Q4 2013 Smartphone OS Results: Is Google Losing Control of the Android Ecosystem?, https://www.abiresearch.com/press/q4-2013smartphone-os-results-is-google-losing-con Accessed 22 April 2014.

[22] Gartner Says Annual Smartphone Sales Surpassed Sales of Feature Phones for the First Time in 2013 http://www.gartner.com/newsroom/id/2665715 Accessed 22 April 2014.

[23] Android developer's guide on sensors http:// developer.android.com/guide/topics/sensors/sensors_overview.html Accessed 22 April 2014. 